\title{
Tenofovir Loaded Poly (Lactide-Co-Glycolide) Nanocapsules: Formulation Optimization by Desirability Functions Approach
}

\author{
Grandhi Srikar ${ }^{\star 1,2}$, Avula Prameela Rani ${ }^{2}$ \\ ${ }^{1}$ School of Pharmacy, Jawaharlal Nehru Technological University Kakinada, Kakinada, Andhra Pradesh, INDIA. \\ ${ }^{2}$ College of Pharmaceutical Sciences, Acharya Nagarjuna University, Guntur, Andhra Pradesh, INDIA.
}

\begin{abstract}
Background: Nanoparticles made of biodegradable polymers are the most effective colloidal drug delivery systems. The in vitro characteristics as well as in vivo presentation of these nanoparticles are majorly influenced by their formulation parameters. Objectives: The principle objective of the work was to optimize various critical formulation parameters by using statistical tools in order to develop poly (lactide-co-glycolide) (PLGA) nanocapsules loaded with tenofovir disoproxil fumarate (TDF) with effective characteristic properties. Methodology: In this work, biodegradable nanocapsules with PLGA for TDF were prepared by modified double emulsification technique. Concentration of PLGA, concentration of Pluronic F-68 in secondary emulsion and concentration of glycerol in external phase were selected as the formulation factors hence, a $3^{3}$ full factorial design in response surface methodology was chosen as an experimental design. The prepared nanocapsules were evaluated for various characteristic properties like particle size, surface morphology, entrapment efficiency, drug release studies and DSC studies. The selected formulation parameters were optimized by a mathematical model called desirability functions approach with the set desirability of high entrapment efficiency, low drug release constant and less particle size. Results and Conclusion: Particle size, entrapment efficiency and drug release constant were found in the ranges of $249.3-376.3 \mathrm{~nm}, 20.95-71.35 \%$ and $0.039-0.184 \mathrm{hr}^{-1}$. These selected formulation parameters were found to have significant (at $p<0.05$ by ANOVA) influence on various characteristics of prepared nanocapsules. The optimized formulation found to have $63.08 \%$ of entrapment efficiency, $284.53 \mathrm{~nm}$ of particle size with zeta potential of $-26.1 \mathrm{mV}$ and $0.054 \mathrm{~h}^{-1}$ of release rate constant.

Key words: Tenofovir, Nanocapsules, Optimization, Response surface methodology, Desirability functions approach.
\end{abstract}

\section{INTRODUCTION}

Polymeric nanoparticles are most effective colloidal drug delivery systems than others like liposomes, niosomes, solid lipid nanoparticles and resealed erythrocytes due to their high physical stability and amendable surface properties. ${ }^{1}$ These can easily diffuse deeper into tissues due to their nano-size and suitable surface properties hence these can be best employed for targeted delivery of drugs directly at the site of action, ${ }^{2}$ thus reducing side effects and also dose of the drugs. Polymeric nanoparticles prepared with biodegradable polymers like PLGA, ${ }^{3}$ poly lactic acid (PLA), ${ }^{4}$ poly
(E-caprolactone), ${ }^{5}$ poly (methyl methacrylate). ${ }^{6}$ are having additional advantages like biocompatibility, biodegradability and low/no toxicity. ${ }^{7}$ The major physicochemical properties viz. entrapment efficiency, particle size, drug release constant and surface charge significantly influence the in vitro as well as in vivo performance of nanoparticles. ${ }^{8}$ These physicochemical properties largely depend on the formulation as well as process parameters of nanoparticles. Hence, optimization of these formulation parameters such as nature and concentration of polymers, surfactants, solvents, stabiliz-
Submission Date: 31-12-2019; Revision Date: 26-02-2020; Accepted Date: 02-05-2020

DOI: 10.5530/ijper.54.2s.79 Correspondence: Mr. Grandhi Srikar Assistant Professor, University College of Pharmaceutical Sciences, Acharya Nagarjuna University, Nagarjuna Nagar-522510, Guntur, Andhra Pradesh, INDIA.

Phone: +919491335467 E-mail: srikar.grandhi@ gmail.com

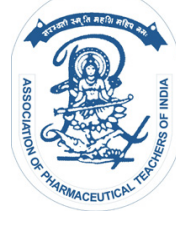

www.ijper.org 
ers used and process parameters such as temperature, stirring speed, in order to obtain nanoparticles with effective properties for their improved performance is of most important area of research.

Various research reports have been published in this area but still there is a large scope for exploration of new methods and optimization of related formulation and process parameters. Some of the works reported earlier were effect of stirring speed, organic phase injection rate, polymer concentration and stabilizer concentration on the polycaprolactone nanoparticles prepared by solvent displacement method reported by Waisudin Badri et al. ${ }^{9}$ effect of organic to aqueous phase volume ratio, surfactant concentration, polymer concentration and type of polymer on biodegradable nanoparticles of paclitaxel prepared by emulsion solvent evaporation method reported by Navneet Sharma et al. ${ }^{10}$ optimization of polymer content, stabilizer concentration, surfactant in primary emulsion and stirring speed on PLGA nanoparticles prepared by w/o/w emulsification using statistical methods reported by Pradipta Sarkar et al. ${ }^{11}$ optimization of surfactant concentration, amount of PLGA and sonication cycles on nanoparticles prepared by emulsion solvent evaporation method reported by Nazimuddin Chishti et al. ${ }^{12}$ But, no literature was reported yet on the influence of external phase properties of secondary emulsion on polymeric nanoparticles prepared by multiple emulsion method.

Response Surface Methodology (RSM), a statistical tool, was adopted in this work in order to obtain meaningful and significant conclusions regarding the influence of various formulation parameters at different levels on the response variables. RSM is a compilation of statistical and mathematical techniques that are useful in optimizing a process/product. RSM is known for its robustness in various applications including designing, developing, optimizing processes and new products as well. ${ }^{13}$ Desirability function approach is the most successful mathematical/statistical approach to optimize the selected formulation and/or process parameters with target of desired values of critical quality characteristics. In this work, we aimed to develop and optimize formulation of PLGA nanoparticles of tenofovir disoproxil fumarate (TDF) prepared by modified multiple emulsification method. Three formulation factors were selected viz. polymer concentration, concentration of stabilizer in secondary emulsion and composition of outer external phase at three levels each were taken as independent variables; three critical quality characteristics of nanoparticles viz. size, entrapment efficiency and drug release constant were selected as responses/dependent variables. The experiment was designed as a $3^{3}$ full factorial design under RSM. The prepared nanoparticles at various combinations of factors according to the design were characterized for the response variables. Later, optimization by desirability function approach was done with the target of less particle size, more entrapment of drug and less drug release constant.

\section{MATERIALS AND METHODS}

\section{Materials}

TDF was procured as gift sample from Hetero Drugs Ltd. Poly (D,L lactide-co-glycolide) (RESOMER RG503H/ PLGA RG503H) was bought from SigmaAldrich, Mumbai. DMEM (Dulbecco's modified Eagles medium), [3-(4,5-dimethylthiazol-2-yl)-2,5-diphenyl tetrazolium bromide] (MTT), trypsin, EDTA Phosphate Buffered Saline (PBS) were acquired from Sigma Chemicals Co. and Fetal Bovine Serum (FBS) was bought from Gibco. $25 \mathrm{~cm}^{2}, 75 \mathrm{~cm}^{2}$ flask and 96 well plated were bought from eppendorf India. Pluronic F-68, SLS, Span 80 and Chloroform were acquired from S.D Fine Chemicals, Mumbai.

\section{Preparation of PLGA Nanocapsules of TDF Experimental design}

In this work, PLGA Nanocapsules of TDF (PLGA-TDFNCs) were aimed to develop by multiple emulsification technique. Three formulation parameters viz. concentration of polymer (A: PLGA RG503H - 50\%, 62.5\% and $75 \%$ ), amount of surfactant (B: Pluronic F-68, $0 \%$, $0.25 \%$ and $0.5 \% \mathrm{w} / \mathrm{v}$ ) in the secondary emulsion and, the external phase in the final emulsion (C: Aqueous glycerol solution, $0 \%, 25 \%$ and $50 \% \mathrm{v} / \mathrm{v}$ of glycerol in water) were selected as the factors and all were taken 3 levels each. So, the experiment was developed as a $3^{3}$ factorial design by using Design Expert software v8.0. All the possible combinations of the factors were taken as a single block with 1 center point. The major characteristics of nanoparticles viz. particle size, entrapment efficiency and drug release constant were taken as dependent variables. Description of the factors and their levels taken was given in Table 1 . The various combinations of the selected factors at taken levels were shown in Table 2.

\section{Preparation of PLGA-TDF-NCs}

PLGA-TDF-NCs were prepared by double emulsification (w/o/w) method reported by Khushwant S. Yadav et al..$^{14}$ after making necessary modifications. The primary internal water phase was prepared by dissolving TDF in a mixture of distilled water and methanol taken 
in the ratio of 4:1. PLGA RG503H was dissolved in chloroform. $2 \mathrm{~mL}$ of aqueous phase was then added drop wise to $10 \mathrm{~mL}$ of chloroform as organic phase under stirring at $12000 \mathrm{rpm}$ for $30 \mathrm{~min}$ to obtain w/o emulsion. Then this w/o emulsion was slowly added

\begin{tabular}{|c|c|c|c|c|}
\hline \multirow[t]{2}{*}{ Factor } & \multirow[t]{2}{*}{ Description } & \multicolumn{3}{|c|}{ Level } \\
\hline & & -1 & 0 & +1 \\
\hline A & $\begin{array}{l}\text { Polymer concentration (\%w/w) } \\
\text { in final weight of nanocapsules }\end{array}$ & 50 & 62.5 & 75 \\
\hline B & $\begin{array}{l}\text { Surfactant concentration } \\
(\% \mathrm{w} / \mathrm{v}) \text { in secondary emulsion }\end{array}$ & 0 & 0.25 & 0.5 \\
\hline C & $\begin{array}{l}\text { Concentration of glycerol } \\
(\% \mathrm{v} / \mathrm{v}) \text { in external phase }\end{array}$ & 0 & 25 & 50 \\
\hline
\end{tabular}

to $20 \mathrm{ml}$ of external phase (distilled water / $25 \% \mathrm{v} / \mathrm{v}$ aqueous glycerol $/ 50 \% \mathrm{v} / \mathrm{v}$ aqueous glycerol) comprising $0.0 \%$ or $0.25 \%$ or $0.5 \% \mathrm{w} / \mathrm{v}$ of Pluronic F- 68 while the mixture was in continuous stirring. The stirring was continued until the chloroform was evaporated. The resulted aqueous nano-dispersion was centrifuged (Sorvall ST 8R, ThermoFisher Scientific) (for $30 \mathrm{~min}$ at $8,000 \mathrm{rpm}$ and $4^{\circ} \mathrm{C}$ to obtain PLGA-TDF-NCs as pellet. This was washed twice with distilled water to take out any unentrapped TDF and the pellet was collected. The pellet was again dispersed in fresh distilled water and freeze-dried (FDB-5502, Operon) for 24hr to yield solid PLGA-TDF-NCs in powder.

\section{Physical characterization studies}

The prepared PLGA-TDF-NCs were studied for various physical characteristics. Differential Scanning

\begin{tabular}{|c|c|c|c|c|c|c|c|c|c|}
\hline \multirow{2}{*}{ S. No. } & \multirow{2}{*}{$\begin{array}{l}\text { Formulation } \\
\text { code }\end{array}$} & \multirow{2}{*}{$\begin{array}{l}\text { Run } \\
\text { order }\end{array}$} & \multicolumn{3}{|c|}{ Factor level } & \multirow{2}{*}{ EE (\%) } & \multirow{2}{*}{ LE (\%) } & \multirow{2}{*}{$\begin{array}{l}\text { Particle size } \\
\text { (nm) }\end{array}$} & \multirow{2}{*}{$\begin{array}{c}k \\
\left(h^{-1}\right)\end{array}$} \\
\hline & & & A & B & C & & & & \\
\hline 1 & $\mathrm{~F} 1$ & 21 & -1 & -1 & -1 & $20.95 \pm 2.12$ & $10.48 \pm 1.06$ & $326.9 \pm 8.3$ & $0.184 \pm 0.02$ \\
\hline 2 & $\mathrm{~F} 2$ & 18 & -1 & 0 & -1 & $30.67 \pm 2.89$ & $15.34 \pm 1.44$ & $318.2 \pm 3.2$ & $0.151 \pm 0.03$ \\
\hline 3 & F3 & 17 & -1 & +1 & -1 & $26.84 \pm 3.21$ & $13.42 \pm 1.61$ & $312.6 \pm 6.5$ & $0.122 \pm 0.04$ \\
\hline 4 & F4 & 16 & -1 & -1 & 0 & $29.36 \pm 4.11$ & $14.68 \pm 2.06$ & $300.2 \pm 4.8$ & $0.145 \pm 0.05$ \\
\hline 5 & F5 & 3 & -1 & 0 & 0 & $45.76 \pm 2.16$ & $22.88 \pm 1.08$ & $290.7 \pm 8.3$ & $0.103 \pm 0.04$ \\
\hline 6 & F6 & 4 & -1 & +1 & 0 & $38.17 \pm 1.19$ & $19.09 \pm 0.60$ & $284.4 \pm 6.1$ & $0.055 \pm 0.01$ \\
\hline 7 & F7 & 13 & -1 & -1 & +1 & $36.58 \pm 5.18$ & $18.29 \pm 2.59$ & $270.1 \pm 2.9$ & $0.117 \pm 0.03$ \\
\hline 8 & F8 & 11 & -1 & 0 & +1 & $58.15 \pm 4.81$ & $29.08 \pm 2.40$ & $258.6 \pm 5.3$ & $0.082 \pm 0.02$ \\
\hline 9 & F9 & 26 & -1 & +1 & +1 & $50.75 \pm 7.12$ & $25.38 \pm 3.56$ & $249.3 \pm 5.1$ & $0.05 \pm 0.02$ \\
\hline 10 & F10 & 22 & 0 & -1 & -1 & $34.45 \pm 1.65$ & $11.22 \pm 0.54$ & $352.2 \pm 7.8$ & $0.165 \pm 0.03$ \\
\hline 11 & F11 & 9 & 0 & 0 & -1 & $39.62 \pm 4.02$ & $12.91 \pm 1.31$ & $343.6 \pm 9.2$ & $0.133 \pm 0.06$ \\
\hline 12 & F12 & 25 & 0 & +1 & -1 & $37.91 \pm 3.52$ & $12.35 \pm 1.14$ & $337.2 \pm 3.4$ & $0.096 \pm 0.02$ \\
\hline 13 & F13 & 14 & 0 & -1 & 0 & $40.25 \pm 6.13$ & $13.11 \pm 1.99$ & $338.5 \pm 7.6$ & $0.119 \pm 0.01$ \\
\hline 14 & F14 & 7 & 0 & 0 & 0 & $56.13 \pm 2.57$ & $18.28 \pm 0.84$ & $330.6 \pm 1.9$ & $0.078 \pm 0.02$ \\
\hline 15 & F14 & 8 & 0 & 0 & 0 & $55.26 \pm 3.14$ & $18.00 \pm 1.02$ & $325.9 \pm 2.5$ & $0.078 \pm 0.02$ \\
\hline 16 & F15 & 2 & 0 & +1 & 0 & $45.65 \pm 4.52$ & $14.87 \pm 1.47$ & $318.7 \pm 10.5$ & $0.059 \pm 0.01$ \\
\hline 17 & F16 & 5 & 0 & -1 & +1 & $51.58 \pm 4.73$ & $16.80 \pm 1.54$ & $308.6 \pm 5.3$ & $0.08 \pm 0.02$ \\
\hline 18 & F17 & 1 & 0 & 0 & +1 & $68.15 \pm 2.59$ & $22.20 \pm 0.84$ & $301.1 \pm 8.6$ & $0.055 \pm 0.02$ \\
\hline 19 & F18 & 19 & 0 & +1 & +1 & $56.62 \pm 1.89$ & $18.44 \pm 0.61$ & $292.6 \pm 5.4$ & $0.041 \pm 0.01$ \\
\hline 20 & F19 & 10 & +1 & -1 & -1 & $29.55 \pm 3.15$ & $7.39 \pm 0.79$ & $376.3 \pm 11.8$ & $0.147 \pm 0.05$ \\
\hline 21 & F20 & 6 & +1 & 0 & -1 & $37.61 \pm 2.86$ & $9.40 \pm 0.71$ & $367.2 \pm 12.4$ & $0.108 \pm 0.04$ \\
\hline 22 & F21 & 15 & +1 & +1 & -1 & $36.25 \pm 4.53$ & $9.06 \pm 1.13$ & $358.4 \pm 7.3$ & $0.073 \pm 0.02$ \\
\hline 23 & F22 & 23 & +1 & -1 & 0 & $43.56 \pm 2.96$ & $10.89 \pm 0.74$ & $349.8 \pm 6.4$ & $0.101 \pm 0.03$ \\
\hline 24 & F23 & 12 & +1 & 0 & 0 & $60.43 \pm 7.15$ & $15.11 \pm 1.79$ & $346.7 \pm 8.9$ & $0.059 \pm 0.02$ \\
\hline 25 & F24 & 27 & +1 & +1 & 0 & $50.95 \pm 3.92$ & $12.74 \pm 0.98$ & $337.4 \pm 1.8$ & $0.048 \pm 0.02$ \\
\hline 26 & F25 & 24 & +1 & -1 & +1 & $56.25 \pm 3.16$ & $14.06 \pm 0.79$ & $331.8 \pm 10.3$ & $0.052 \pm 0.01$ \\
\hline 27 & F26 & 28 & +1 & 0 & +1 & $71.35 \pm 4.55$ & $17.84 \pm 1.14$ & $317.5 \pm 12.1$ & $0.046 \pm 0.01$ \\
\hline 28 & F27 & 20 & +1 & +1 & +1 & $60.35 \pm 3.67$ & $15.09 \pm 0.92$ & $307.9 \pm 6.3$ & $0.039 \pm 0.02$ \\
\hline
\end{tabular}

*results expressed as average \pm standard deviation for $n=3$ 
Calorimetry (DSC) (Schimadzu DSC-60) was done in order to check the physical state (amorphous/crystalline) of the drug. ${ }^{15}$ The surface morphology including particle shape was studied by using Transmission Electron Microscope (TEM) (Tecnai G2-30). Size and surface charge were determined by dynamic light scattering principle employing ZetaSizer Nano-ZS90.

\section{Entrapment Efficiency and Drug Loading}

The nano-suspension of PLGA-TDF-NCs obtained after preparation was kept for centrifugation for a period of $30 \mathrm{~min}$ at $8,000 \mathrm{rpm}$ and at a temperature of $4^{\circ} \mathrm{C}$. Supernatant and solid-pellet were separated. The pellet was carefully washed with water over a filtration medium to remove the unentrapped drug. The washings were mixed with the supernatant and spectrophotometrically (Evolution 201, ThermoFisher Scientific) analyzed at $\lambda_{\max }$ of $260 \mathrm{~nm}$ to know the unentrapped amount of TDF from which the entrapped amount of TDF was quantified. The following formulae were used to obtain drug entrapment efficiency and drug loading

$$
\text { Entrapment Efficiency }(\%)=\frac{\begin{array}{l}
\text { amount of drug } \\
\text { entrapped }
\end{array}}{\begin{array}{l}
\text { theoretical amount of drug in } \\
\text { nanocapsules taken }
\end{array}} \times 100
$$$$
\text { Drug loading }(\%)=\frac{\text { amount of drug entrapped }}{\text { total amount of drug and polymer taken }} \times 100
$$

\section{In vitro Drug Release Studies}

In vitro drug release studies were performed employing dialysis bag method as reported by Bohrey $\mathrm{S}$ et al. ${ }^{16}$ and Guptha $\mathrm{N}$ et al. ${ }^{17}$ using dialysis membrane (Dialysis Membrane-110; Molecular weight cut off between 12,000 to 14,000; HiMedia Lab. Pvt. Ltd., Mumbai) and $0.1 \mathrm{~N} \mathrm{HCl}$ as buffer. The amount of TDF released was determined using UV-Visible spectrophotometer at $260 \mathrm{~nm}$. The data obtained were treated using various kinetic models to determine the kinetics and mechanism of drug release.

\section{Experimental Design Validation and ANOVA}

According to the selected design, $3^{3}$ factorial design under response surface methodology, all the possible 27 combinations of the factors were taken as a single block with one center point (so, 28 runs). The obtained values of response variables of all formulations of PLGA-TDF- were statistically treated using polynomial quadratic model of response surface methodology without need of transformation. Linear model represents the effect of factors on a response in linear regression only though the actual effect might be non-linear. But, the quadratic model expresses whether the effect of factors on a response is linear or nonlinear. The selected experimental design was validated for its fit into this polynomial quadratic model by employing ANOVA and also by plotting normal plot of residuals as well as predicted versus actual plot for every response.

\section{Optimization by Desirability Functions Approach}

Desirability function approach was adopted in this study to optimize the factors of this design to achieve the set goals of all the three response variables in a single formulation of PLGA-TDF-NCs of TDF. The goals were set as entrapment efficiency to its maximum and, particle size and release rate constant to their minimum. This optimization was performed through desirability function approach by using the Design Expert software. In this method, every response $\left(y_{i}\right)$ is taken as individual desirability function $\left(\mathrm{d}_{\mathrm{i}}\right)$ which fluctuates over the range of 0 to 1 . The $d_{i}$ is taken as either one when the $y_{i}$ is at its goal or taken as zero when the $y_{\mathrm{i}}$ is outside the acceptable area, so that the independent variables (factors) are selected to maximize the desirability. If the target (T) for any response is to maximize it, then the desirability is calculated from

$$
\mathrm{d}_{\mathrm{i}}=[(\mathrm{y}-\mathrm{L}) /(\mathrm{T}-\mathrm{L})]^{\mathrm{T}}
$$

If the target ( $T$ ) for any response is to minimize it, then the desirability is calculated from

$$
\mathrm{d}_{\mathrm{i}}=[(\mathrm{U}-\mathrm{y}) /(\mathrm{U}-\mathrm{T})]^{\mathrm{r}}
$$

where, $\mathrm{U}$ and $\mathrm{L}$ are upper and lower limits of the response variable respectively. In this study, linear desirability function approach was considered so that $r$ was taken as one. ${ }^{13,18}$

\section{In vitro Cytotoxicity Studies}

The toxicity of PLGA-TDF-NCs was evaluated by MTT assay on HeLa cell lines, which were purchased from NCCS, Pune. Maintenance of the cells and procedure used for determining cytotoxicity were as reported by Venkanna A et al. ${ }^{19}$ The treated cells were incubated with different concentrations of the optimized PLGATDF-NCs in wells in 96 plates for $48 \mathrm{hr}$. Then the test solution was replaced with fresh media of MTT solution $(0.5 \mathrm{mg} / \mathrm{mL})$ and incubated at $37^{\circ} \mathrm{C}$ for $3 \mathrm{hr}$. The color changed to purple due to the reduction of MTT into formazan by the active mitochondria of live cells. The color intensity/ optical density (O.D) was measured at $570 \mathrm{~nm}$ on a microplate reader (Varioskan ${ }^{\mathrm{TM}}$ LUX, ThermoFisher Scientific). The cells untreated with the 
sample were considered as control. The below equation was used to calculate percentage inhibition

$$
\% \text { Inhibition }=\frac{\text { O.D of Control }- \text { O.D of Treatment }}{\text { O.D of Control }} \times 100
$$

\section{RESULTS AND DISCUSSION}

\section{Experimental Design}

Three level full factorial design of response surface methodology was employed in this work to investigate the influence of the selected formulation parameters as independent factors on the characteristics of the PLGA-TDF-NCs as response variables. Full factorial designs are useful in elucidating quadratic effects of the factors on the responses. The results of responses of all the formulations were statistically treated using polynomial quadratic model. The obtained equations for the selected responses were

$$
\begin{array}{cl}
\mathrm{EE} & =55.43+6.06 * \mathrm{~A}+3.39 * \mathrm{~B}+12.00 * \mathrm{C}- \\
& 0.89 * \mathrm{AB}+1.46 * \mathrm{AC}+0.61 * \mathrm{BC}-4.27 * \mathrm{~A}^{2} \\
& -10.52 * \mathrm{~B}^{2}-0.92 * \mathrm{C}^{2} \\
\text { Particle size } & =+326.76+26.78 * \mathrm{~A}-8.66 * \mathrm{~B}-25.28 * \mathrm{C} \\
& -0.27 * \mathrm{AB}+2.92 * \mathrm{AC}-1.12 * \mathrm{BC}- \\
& 7.80 * \mathrm{~A}^{2}+0.35 * \mathrm{~B}^{2}-3.45 * \mathrm{C}^{2} \\
\mathrm{k} \quad & 0.081-0.019 * \mathrm{~A}-0.029 * \mathrm{~B}-0.034^{*} \mathrm{C} \\
& +0.007 * \mathrm{AB}+0.001 * \mathrm{AC}+0.007 * \mathrm{BC}+ \\
& 0.002 * \mathrm{~A}^{2}+0.004 * \mathrm{~B}^{2}+0.012 * \mathrm{C}^{2}
\end{array}
$$

From the equations, it was understood that the entrapment efficiency was positively affected (increasing upon increasing the level of factor) by all the three selected factors; particle size was affected positively by factor A and negatively (decreasing upon increasing the level of factor) by both factors $\mathrm{B}$ and $\mathrm{C}$; and drug release constant was negatively affected by by all the three selected factors.

\section{Transmission Electron Microscopy (TEM)}

The surface morphology of PLGA-TDF-NCs was studied by TEM and the images were shown in Figure 1. These images depicted that the PLGA-TDF-NCs were closely spherical and had uniform surface without any dents or protrusions.

\section{Differential Scanning Calorimetry (DSC)}

DSC spectra of pure TDF, pure polymer PLGA and PLGA-TDF-NCs were shown in Figure 2. The spectrum for pure TDF indicated a sharp endotherm at $118.1^{\circ} \mathrm{C}$ matching to its melting point which is near $115^{\circ} \mathrm{C}^{20} \mathrm{But}$ this peak was not observed in the spectrum of PLGA-TDF-NCs, thus designated that the TDF was not in crystalline state in the PLGA-TDF-NCs instead

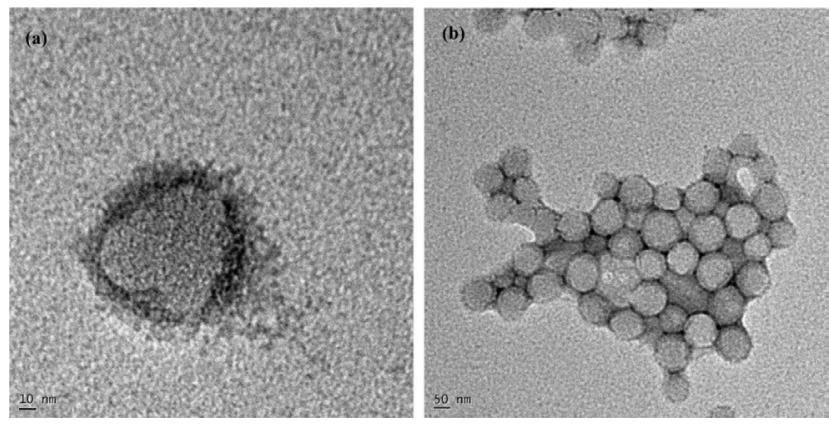

Figure 1: TEM images of PLGA-TDF-NCs of the optimized formulation.

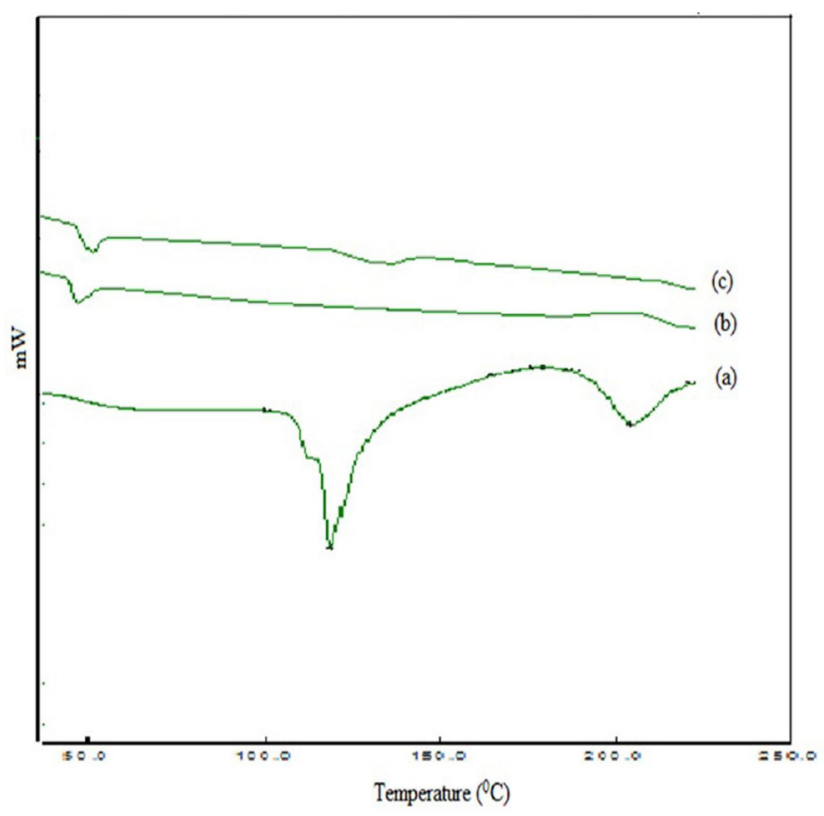

Figure 2: DSC spectra of (a) Pure TDF; (b) Pure PLGA RG503H; and (c) PLGA-TDF-NCs.

the TDF might be in amorphous state or in molecular dispersion form. ${ }^{21}$ This could be due to the way of assimilation of drug i.e. in its solution form into the PLGA-TDF-NCs during formation. In case of PLGA, an endotherm at $48.1^{\circ} \mathrm{C}$ was observed in its pure spectrum agreeing to its glass transition temperature $(\mathrm{Tg})$. But the spectrum of PLGA-TDF-NCs showed an endotherm at $52.3^{\circ} \mathrm{C}$, thus indicating the $\mathrm{Tg}$ of PLGA was increased which could be due to its quick solidification upon evaporation of chloroform during the process. $^{22}$

\section{Particle Size}

Particle size values of all formulations of PLGA-TDFNCs were found to be in the range of $249.3-376.3 \mathrm{~nm}$ and were given in Table 2. Effect of the factors on particle size was showed in Figure 3(a). Increase in 


\begin{tabular}{|c|c|c|c|c|c|c|}
\hline \multicolumn{2}{|c|}{ Table 3: Results of ANOVA for response surface quadratic model for the response particle size. } \\
\hline Source & Sum of squares & $\begin{array}{c}\text { Degrees of } \\
\text { freedom }\end{array}$ & $\begin{array}{c}\text { Mean sum of } \\
\text { squares }\end{array}$ & F value & \multicolumn{1}{c|}{$\boldsymbol{p \text { -Value }}$} & Inference $^{\text {a }}$ \\
\hline Model & 26370.51 & 9 & 2930.06 & 269.01 & $<0.0001$ & Significant \\
\hline $\mathrm{A}^{\mathrm{b}}$ & 12906.89 & 1 & 12906.89 & 1184.97 & $<0.0001$ & Significant \\
\hline $\mathrm{B}^{\mathrm{c}}$ & 1350.27 & 1 & 1350.27 & 123.97 & $<0.0001$ & Significant \\
\hline $\mathrm{C}^{\mathrm{d}}$ & 11506.45 & 1 & 11506.45 & 1056.40 & $<0.0001$ & Significant \\
\hline $\mathrm{AB}$ & 0.91 & 1 & 0.91 & 0.083 & 0.7761 & Not significant \\
\hline $\mathrm{AC}$ & 102.08 & 1 & 102.08 & 9.37 & 0.0067 & Significant \\
\hline $\mathrm{BC}$ & 15.19 & 1 & 15.19 & 1.39 & 0.2530 & Not significant \\
\hline $\mathrm{A}^{2}$ & 388.25 & 1 & 388.25 & 35.64 & $<0.0001$ & Significant \\
\hline $\mathrm{B}^{2}$ & 0.76 & 1 & 0.76 & 0.07 & 0.7942 & Not significant \\
\hline $\mathrm{C}^{2}$ & 76.05 & 1 & 76.05 & 6.98 & 0.0166 & Significant \\
\hline Residual & 196.06 & 18 & 10.89 & -- & - & -- \\
\hline Lack of Fit & 185.01 & 17 & 10.88 & 0.99 & 0.6721 & Not significant \\
\hline
\end{tabular}

Note: ${ }^{a} p$-Value less than 0.05 indicates model terms are significant; ${ }^{b}$ Polymer concentration $(\% \mathrm{w} / \mathrm{w}) ;{ }^{\mathrm{c}}$ Surfactant concentration in secondary emulsion $(\% \mathrm{w} / \mathrm{v})$; ${ }^{\mathrm{d}}$ Concentration of glycerol in external phase $(\% \mathrm{v} / \mathrm{v})$

polymer concentration resulted in increase of particle size. This might be because of the fact that the polymer deposits on the surface of core material upon solvent removal $^{23}$ and hence at higher polymer levels more amount of polymer might deposit around the dispersed phase globules thus resulting in bigger particles. Shearing efficiency might also be diminished at higher viscosities that might lead to formation of large particles. ${ }^{24}$ The particle size of nanoparticles containing surfactant was found to be less than those prepared without surfactant. This might be because of the potential of surfactant to decrease the interfacial tension henceforth the interfacial free energy, such that an emulsion with smaller particle size (or higher interfacial area) could be obtained with greater stability. These results were correlated with those reported by Y. Krishnamachari et al. ${ }^{25}$ and Gupta et al. ${ }^{26}$ Particle size was observed to be more in water as the external phase of secondary emulsion than that with aq. glycerol $25 \%$ and $50 \% \mathrm{v} / \mathrm{v}$. The increased viscosity of the outer phase upon increase in concentration of glycerol, would resist the aggregation of globules from primary emulsion. The effect of all the factors on particle size was found to be significant at $p<0.05$ (Table 3).

\section{Zeta Potential}

Zeta potential values of all formulations of PLGATDF-NCs were given in Table 2 and found to be varied from a minimum of $-21.6 \mathrm{mV}$ to $-27.6 \mathrm{mV}$ with the average zeta potential of $-24.25 \mathrm{mV}$. These values showed that none of the three factors influenced as the zeta potential might depend majorly on the chemical nature of polymer that forms the outer surface of the nanocapsules. The polymer employed in all the
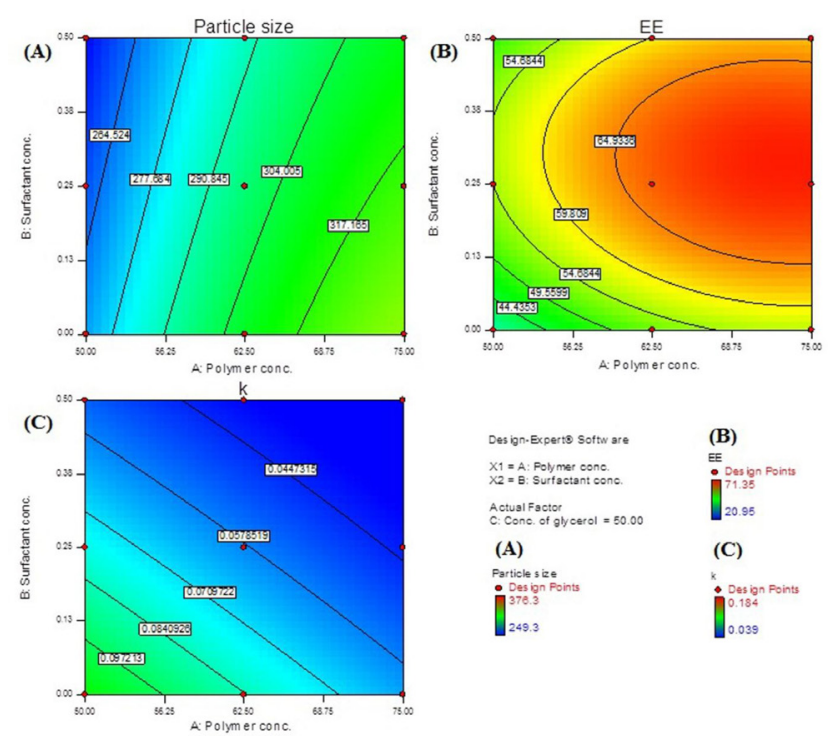

Figure 3: Contour plots showing influence of polymer concentration (factor $A$ ) and surfactant concentration (factor $B$ ) at $50 \% \mathrm{v} / \mathrm{v}$ glycerol in the external aqueous phase on the responses (A) Particle size; (B) Entrapment efficiency; (C) Drug release rate constant.

formulations here was PLGA RG503H and the negative zeta potential of PLGA-TDF-NCs was due to the free carboxylic acid end groups on the polymer. ${ }^{27}$

\section{Entrapment Efficiency}

Entrapment efficiency values of all formulations of PLGA-TDF-NCs were given in Table 2 and were observed to be varied from $20.95 \%$ to $71.35 \%$. Effect of the selected factors on entrapment efficiency was showed in Figure 3(b). The influence of concentration of polymer was clearly evident that the entrapment efficiency was observed to be increased as the polymer 
concentration was increased. This might be because of the fact that high amount of polymer could bind the drug more tightly so that leakage of the drug would be minimized. Higher amounts of polymer also lead to increase in viscosity so that the diffusion of drug from innermost aqueous phase to organic phase could be minimized resulting in higher entrapment efficiency. ${ }^{25}$ Also, at higher polymer levels, the increase in particle size minimized the escape of the drug by diffusion out of the nanocapsules through reduced surface area and increased path length and hence the entrapment efficiency could be increased ${ }^{28}$ (Görner et al. 1999). The influence of surfactant concentration was interesting as upon its increase from $0.0 \%$ to $0.25 \%$, the entrapment efficiency was increased but a further increase to $0.5 \%$ lead to decrease in the entrapment efficiency. The initial increase could be due to the stabilization of the emulsion because of the surfactant and thus abridged leakage of the drug. But, the drug would be diffused out of the nanocapsules and would be micellar solubilized in the external aqueous phase upon a further increase in surfactant concentration. ${ }^{29}$ The increase in entrapment efficiency upon increase in glycerol concentration in the external phase of the secondary emulsion would be attributed to the increase in viscosity as well density the external phase. Since, the viscosity would reduce the diffusion according to Stokes - Einstein equation, ${ }^{30}$ leakage of the drug into external phase would be reduced and lead to increase in the entrapment efficiency. Effect of all the three factors was found to be significant at $p<0.05$ (shown in Table 4).

The maximum entrapment efficiency was found to be only $71.35 \%$, which could be due to highly water soluble nature of the drug TDF that might leach to some extent into the external aqueous phase. However this entrapment efficiency value for the prepared PLGATDF-ACNs was found to be more than those reported by other authors ${ }^{31,32}$ in the case of similar high water soluble drugs signifying that both PLGA polymer and ACNs technique were capable of loading water soluble drugs into nanocapsules.

\section{In vitro Drug Release Studies}

The drug release rate constant values of all formulations of PLGA-TDF-NCs were given in Table 2 and were observed to be varied from $0.039 \mathrm{hr}^{-1}$ to $0.184 \mathrm{hr}^{-1}$. Effect of the three factors was showed in the Figure 3(c). The effect of amount of polymer was significant on the release of the drug from the prepared PLGA-TDF-NCs that upon increasing the amount of polymer, the drug release was found to be decreased. This might be due to the increase in particle size, which reduces the dissolution rate, upon increase in the amount of polymer. As the particle size was increased, the mean surface area would be decreased and the path length for diffusion would be increased, which might combinely result in decreased drug release rate. Effect of concentration of surfactant in secondary emulsion was significant that upon increase in its concentration, the drug release was observed to be decreased. As the amount of surfactant increases, the outer volatile organic phase (Chloroform) of primary emulsion might interact more with the external aqueous phase that lead to a decrease in the rate of evaporation which finally resulted in nanocapsules with a more rigid membrane ${ }^{10,33}$ so that the drug release rate got decreased. The concentration of glycerol in the

\begin{tabular}{|c|c|c|c|c|c|c|}
\hline Source & $\begin{array}{l}\text { Sum of } \\
\text { squares }\end{array}$ & $\begin{array}{l}\text { Degrees of } \\
\text { freedom }\end{array}$ & $\begin{array}{c}\text { Mean sum of } \\
\text { squares }\end{array}$ & $F$ value & $p$-Value & Inference $^{a}$ \\
\hline Model & 4381.61 & 9 & 486.85 & 56.00 & $<0.0001$ & Significant \\
\hline$A^{b}$ & 660.90 & 1 & 660.90 & 76.02 & $<0.0001$ & Significant \\
\hline $\mathrm{B}^{\mathrm{c}}$ & 206.45 & 1 & 206.45 & 23.75 & 0.0001 & Significant \\
\hline$C^{d}$ & 2590.32 & 1 & 2590.32 & 297.95 & $<0.0001$ & Significant \\
\hline$A B$ & 9.51 & 1 & 9.51 & 1.09 & 0.3096 & Not significant \\
\hline$A C$ & 25.58 & 1 & 25.58 & 2.94 & 0.1035 & Not significant \\
\hline $\mathrm{BC}$ & 4.39 & 1 & 4.39 & 0.51 & 0.4863 & Not significant \\
\hline$A^{2}$ & 116.22 & 1 & 116.22 & 13.37 & 0.0018 & Significant \\
\hline $\mathrm{B}^{2}$ & 705.72 & 1 & 705.72 & 81.18 & $<0.0001$ & Significant \\
\hline $\mathrm{C}^{2}$ & 5.39 & 1 & 5.39 & 0.62 & 0.4412 & Not significant \\
\hline Residual & 156.49 & 18 & 8.69 & -- & -- & -- \\
\hline Lack of Fit & 156.11 & 17 & 9.18 & 24.26 & 0.1585 & Not significant \\
\hline
\end{tabular}

Note: a ${ }^{\mathrm{p}}$-Value less than 0.05 indicates model terms are significant; ${ }^{\mathrm{b}}$ Polymer concentration $(\% \mathrm{w} / \mathrm{w}){ }_{i}{ }^{\mathrm{c}}$ Surfactant concentration in secondary emulsion $(\% \mathrm{w} / \mathrm{v})$; ${ }^{\mathrm{d}}$ Concentration of glycerol in external phase $(\% \mathrm{v} / \mathrm{v})$ 


\begin{tabular}{|c|c|c|c|c|c|c|}
\hline Source & $\begin{array}{l}\text { Sum of } \\
\text { squares }\end{array}$ & $\begin{array}{l}\text { Degrees of } \\
\text { freedom }\end{array}$ & $\begin{array}{c}\text { Mean sum of } \\
\text { squares }\end{array}$ & F value & $p$-Value & Inference $^{a}$ \\
\hline Model & 0.045 & 9 & $5.011 \times 10^{-3}$ & 78.09 & $<0.0001$ & Significant \\
\hline$A^{b}$ & $6.272 \times 10^{-3}$ & 1 & $6.272 \times 10^{-3}$ & 97.74 & $<0.0001$ & Significant \\
\hline $\mathrm{B}^{\mathrm{c}}$ & 0.015 & 1 & 0.015 & 240.45 & $<0.0001$ & Significant \\
\hline$C^{d}$ & 0.021 & 1 & 0.021 & 329.59 & $<0.0001$ & Significant \\
\hline$A B$ & $5.201 \times 10^{-4}$ & 1 & $5.201 \times 10^{-4}$ & 8.10 & 0.0107 & Significant \\
\hline $\mathrm{AC}$ & $2.408 \times 10^{-5}$ & 1 & $2.408 \times 10^{-5}$ & 0.38 & 0.5478 & Not significant \\
\hline $\mathrm{BC}$ & $6.163 \times 10^{-4}$ & 1 & $6.163 \times 10^{-4}$ & 9.60 & 0.0062 & Significant \\
\hline$A^{2}$ & $2.550 \times 10^{-5}$ & 1 & $2.550 \times 10^{-5}$ & 0.40 & 0.5364 & Not significant \\
\hline $\mathrm{B}^{2}$ & $9.368 \times 10^{-5}$ & 1 & $9.368 \times 10^{-5}$ & 1.46 & 0.2426 & Not significant \\
\hline $\mathrm{C}^{2}$ & $8.927 \times 10^{-4}$ & 1 & $8.927 \times 10^{-4}$ & 13.91 & 0.0015 & Significant \\
\hline Residual & $1.155 \times 10^{-3}$ & 18 & $6.417 \times 10^{-5}$ & -- & -- & -- \\
\hline Lack of Fit & $1.155 \times 10^{-3}$ & 17 & $6.794 \times 10^{-5}$ & -- & $<0.0001$ & Not significant \\
\hline
\end{tabular}

Note: ${ }^{a} p$-Value less than 0.05 indicates model terms are significant; ${ }^{b}$ Polymer concentration (\%w/w); ${ }^{c}$ Surfactant concentration in secondary emulsion (\%w/v); ${ }^{\mathrm{d}}$ Concentration of glycerol in external phase $(\% \mathrm{v} / \mathrm{v})$

external phase significantly influenced the release rate of TDF from the PLGA-TDF-NCs as the drug release was observed to be decreased upon increase in the concentration of glycerol. This might be because of the increase in viscosity at higher concentrations of glycerol that might decrease the evaporation of chloroform. The nanocapsules obtained by slow evaporation rates might have the polymer membrane rigid with less porosity, ${ }^{33}$ which could reduce the rate of drug release from them. Effects of all the three factors was found to be significant at $p<0.05$ (shown in Table 5). All the formulations showed first - order kinetics of drug release and non - Fickian diffusion type of mechanism of drug release.

\section{Experimental Design Validation and ANOVA}

The normal plot of residuals, shown in Figure 4 (a), was obtained as a straight line which indicated the residuals followed normal distribution and thus the response values did not require any transformation for further analysis. The predicted versus actual plot, shown in Figure 4(b), indicated that most of the actual values obtained were close to the values predicted by the design, which confirmed any transformation of responses was not necessary. The results of ANOVA were shown in Tables 3-5. The model $F$ value for every response indicated that the model was significant designating that the response surface quadratic model selected was appropriate for the experimental design adapted in this work. This was further evidenced by insignificant lack-of-fit values. The lack-of-fit means the selected model does not fit to the experimental design which further suggests that change of model or experimental design. But, as the obtained lack-of-fit
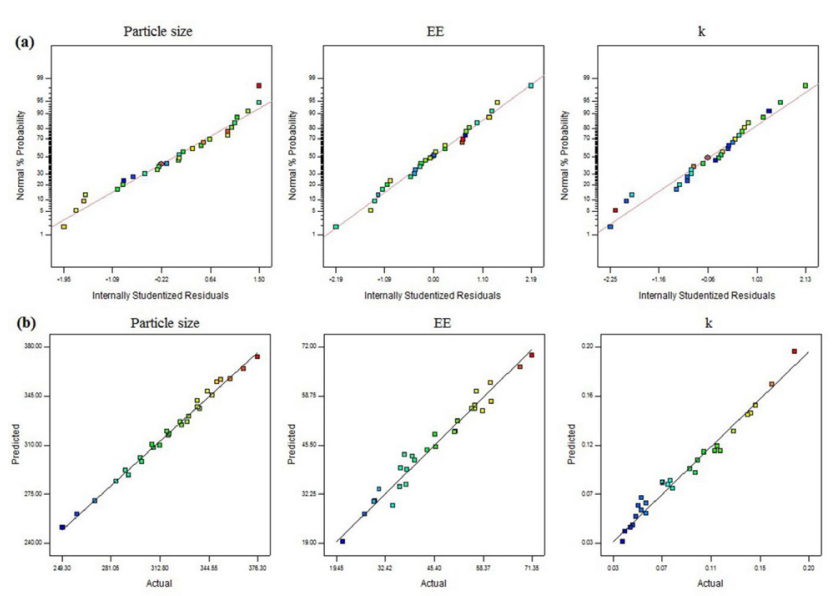

Figure 4: (a) Normal plot of residuals of all the three responses; (b) Predicted versus actual plots of all the three responses.

values were insignificant, the selected quadratic model was fit for the chosen experimental design.

\section{Optimization by Desirability Function Approach}

High entrapment efficiency (decrease the quantity of formulation per dose), less particle size (increases tissue permeability) and low drug release rate constant (prolongs the duration of action) are always desirable for improved in vitro as well as in vivo performance of nanoparticles. The results so far indicated that influence of a factor on all the responses was not as desirable which could be evident from the effect of polymer concentration on the responses. The increase in polymer concentration resulted in increased in entrapment efficiency and decreased drug release rate constant which are desired but it also caused increase 


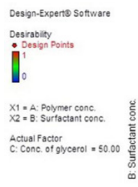

(a)
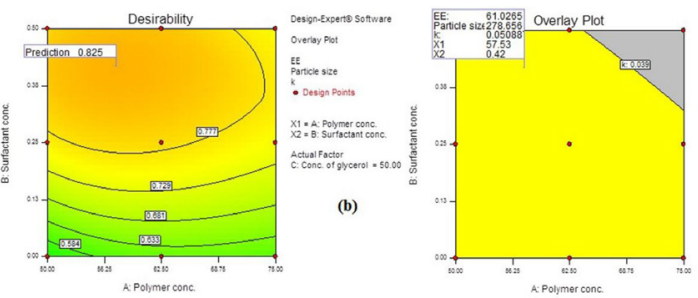

Figure 5: (a) Desirability plot; (b) Overlay plot showing the optimized values of the factors and predicted values of the responses.

in particle size which is not desirable. Hence, optimization of the factors should be done for desirable results of all the characteristics. Levels of the selected independent variables were optimized with the desirability of PLGA-TDF-NCs having less size, high entrapment and low drug release constant. Desirability was set as the entrapment efficiency to maximum; particle size to minimum; and drug release constant to minimum. Among the various solutions suggested by the software for the set desirability, one solution with maximum desirability of 0.825 (shown in Figure 5(a)) at a combination of the factors as Factor A at $57.69 \% \mathrm{w} / \mathrm{w}$, Factor B at $0.42 \% \mathrm{w} / \mathrm{v}$ and Factor $\mathrm{C}$ at $50.00 \% \mathrm{v} / \mathrm{v}$ was considered where the response variables as shown by the software were $61.16 \%$ of entrapment efficiency, $279.12 \mathrm{~nm}$ of particle size and $0.051 \mathrm{~h}^{-1}$ of release rate constant as Figure 5(b). A formulation of PLGA-TDFNCs was prepared by taking this optimized combination of independent variables and characterized for the response variables. The obtained results were found to be $63.08 \%$ of entrapment efficiency, $284.53 \mathrm{~nm}$ of particle size with zeta potential of $-26.1 \mathrm{mV}$ and $0.054 \mathrm{~h}^{-1}$ of release rate constant which were correlated to those given by the design. Hence, this combination was considered as the optimized levels of the studied independent variables.

\section{In vitro Cytotoxicity Studies}

In vitro cytotoxicity study was performed by MTT assay on HeLa cell lines for the PLGA-TDF-NCs of optimized formulation at five different concentrations ranging from $5-200 \mu \mathrm{g} / \mathrm{mL}$. The pictures of cells after the treatment were shown in Figure 6(a) - 6(e). From the obtained $\%$ viability at these concentrations, the viability plot as shown in Figure 6(f), was made by taking concentration on $\mathrm{x}$-axis and $\%$ viability on $y$-axis and obtained its linear regression equation. The half maximal inhibitory concentration $\left(\mathrm{IC}_{50}\right)$ is the concentration of the test compound required to inhibit the growth of $50 \%$ of cells. ${ }^{34} \mathrm{The}^{\mathrm{IC}} \mathrm{C}_{50}$ of PLGA-TDF-NCs was found to be $236.5 \pm 17.4 \mu \mathrm{g} / \mathrm{mL}$. This high $\mathrm{IC}_{50}$

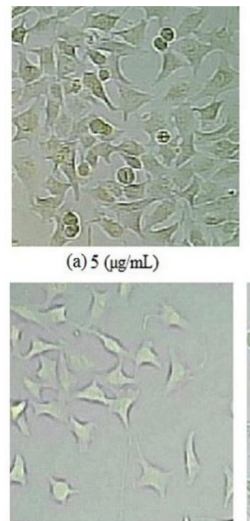

(d) $100(\mu \mathrm{g} / \mathrm{mL})$

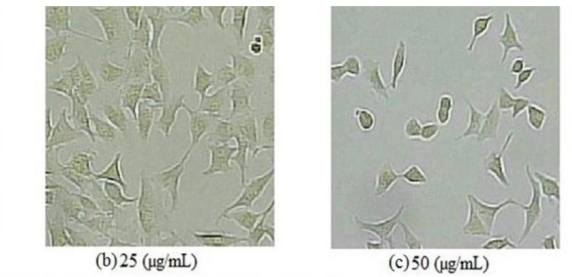

(c) $50(\mu \mathrm{g} / \mathrm{mL})$

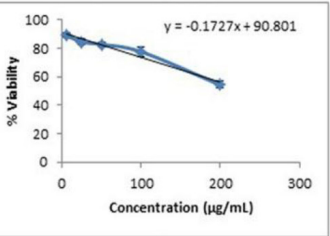

(f)
Figure 6: MTT assay images showing (a) - (e) The intensity of cells at different concentrations of PLGA-TDF-NCs; (f) Plot of concentration versus $\%$ viability.

value indicated that either TDF or PLGA RG503H in the PLGA-TDF-NCs is not toxic and relatively safe.

\section{CONCLUSION}

The principle objective of this study was to optimize formulation of PLGA nanocapsules of TDF for improved in vitro as well as in vivo performance. PLGA-TDF-NCs were prepared by modified double emulsion (w/o/w) technique as a $3^{3}$ full factorial design under response surface methodology. The influence of all the selected formulation parameters on all the response variables and also the selected response surface quadratic model were found to be significant at $p<0.05$ by ANOVA studies. Optimization was performed by desirability functions approach which resulted in PLGA-TDF-NCs with entrapment efficiency of $63.08 \%$; particle size of $284.53 \mathrm{~nm}$ with zeta potential of $-26.1 \mathrm{mV}$ and $0.054 \mathrm{~h}^{-1}$ of release rate constant at polymer concentration of $57.69 \% \mathrm{w} / \mathrm{w}$; at surfactant concentration in secondary emulsion of $0.42 \% \mathrm{w} / \mathrm{v}$; and at $50.00 \% \mathrm{v} / \mathrm{v}$ of glycerol in water as external phase. Thus, PLGA nanocapsules of TDF with effective characteristic properties were developed by statistical optimization and the set objective was achieved.

\section{ACKNOWLEDGEMENT}

The authors are acknowledged to the authorities of Jawaharlal Nehru Technological University Kakinada (JNTUK), Kakinada and Acharya Nagarjuna University, Guntur for their constant support throughout the work.

\section{CONFLICT OF INTEREST}

The authors declare that there are no conflicts of interest. 


\section{ABBREVIATIONS}

ANOVA: Analysis of Variance; DMEM: Dulbecco's modified Eagles medium; DMSO: Dimethyl sulphoxide; DoE: Design of Experiments; DSC: Differential Scanning Calorimetry; EE: Entrapment efficiency; FBS: Fetal Bovine Serum; IC $_{50}$ : Half maximal inhibitory concentration; LE: Loading efficiency; MTT: [3-(4,5-dimethylthiazol-2-yl)-2,5-diphenyl tetrazolium bromide]; O.D.: Optical Density; PBS: Phosphate Buffered Saline; PLA: Poly lactic acid; PLGA: Poly (lactide-co-glycolide); PLGA-TDF-NCs: PLGA Nanocapsules of TDF; RSM: Response Surface Methodology; SLS: Sodium lauryl sulphate; TDF: Tenofovir Disoproxil Fumarate; TEM: Transmission Electron Microscopy.

\section{REFERENCES}

1. Barratt GM. Therapeutic applications of colloidal drug carriers. Pharm Sci Technolo Today. 2000;3(5):163-71.

2. Li X, Wu Q, Chen Z, Gong X, Lin X. Preparation, characterization and controlled release of liver-targeting nanoparticles from the amphiphilic random copolymer. Polymer. 2008;49(22):4769-75.

3. Guo LY, Yan SZ, Li Q, Xu Q, Lin X, Qi SS, et al. Poly (lactic-co-glycolic) acid nanoparticles improve oral bioavailability of hypocrellin A in rat. RSC Adv. 2017;7(67):42073-82.

4. DaLuz CM, Boyles MS, Falagan-Lotsch P, Pereira MR, Tutumi HR, DeOliveira $\mathrm{SE}$, et al. Poly-lactic acid nanoparticles (PLA-NP) promote physiological modifications in lung epithelial cells and are internalized by clathrin-coated pits and lipid rafts. J Nanobiotechnology. 2017;15(1):11.

5. Ribeiro AF, DeOliveira RRL, Cabral LM, DeSousa VP. Poly $\varepsilon$-caprolactone nanoparticles loaded with Uncaria tomentosa extract: Preparation, characterization and optimization using the Box-Behnken design. Int $\mathrm{J}$ Nanomed. 2013;8(1):431-42.

6. Gyergyek S, Pahovnik D, Žagar E, Mertelj A, Beković M, Jagodič M, et al. Nanocomposites comprised of homogeneously dispersed magnetic ironoxide nanoparticles and poly (methyl methacrylate). Beilstein J Nanotechnol. 2018;9(1):1613-22.

7. Soppimath KS, Aminabhavi TM, Kulkarni AR, Rudzinski WE. Biodegradable polymeric nanoparticles as drug delivery devices. J Control Release. 2001;70(1-2):1-20.

8. Crucho $\mathrm{Cl}$, Barros MT. Polymeric nanoparticles: A study on the preparation variables and characterization methods. Mater Sci Eng C. 2017;80:771-84.

9. Badri W, Miladi K, Nazari QA, Fessi H, Elaissari A. Effect of process and formulation parameters on polycaprolactone nanoparticles prepared by solvent displacement. Colloids and Surfaces A: Physicochem Eng Aspects. 2017;516:238-44.

10. Sharma N, Madan P, Lin S. Effect of process and formulation variables on the preparation of parenteral paclitaxel-loaded biodegradable polymeric nanoparticles: A co-surfactant study. Asian J Pharm Sci. 2016;11(3):404-16.

11. Sarkar P, Bhattacharya S, Pal TK. Application of statistical design to evaluate critical process parameters and optimize formulation technique of polymeric nanoparticles. R Soc Open Sci. 2019;6(7):190896.

12. Chishti N, Jagwani S, Dhamecha D, Sunil JS, Dehghan MH. Preparation, optimization and in vivo evaluation of nanoparticle-based formulation for pulmonary delivery of anticancer drug. Medicina. 2019;55(6):294.

13. Myers RH, Montgomery DC, Anderson-Cook CM. Response surface methodology: process and product optimization using designed experiments. $3^{\text {rd }}$ ed. New Jersey: John Wiley and Sons. 2009.

14. Yadav KS, Sawant KK. Modified nanoprecipitation method for preparation of cytarabine-loaded PLGA nanoparticles. AAPS Pharm Sci Tech. 2010;11(3):1456-65.
15. Mainardes RM, Gremião MP, Evangelista RC. Thermoanalytical study of praziquantel-loaded PLGA nanoparticles. Braz J Pharm Sci. 2006;42(4):523-30.

16. Bohrey S, Chourasiya V, Pandey A. Polymeric nanoparticles containing diazepam: preparation, optimization, characterization, in-vitro drug release and release kinetic study. Nano Converg. 2016;3(1):3.

17. Gupta N, Rajera R, Nagpal M, Arora S. Primaquine loaded chitosan nanoparticles for liver targeting. Pharm Nanotechnol. 2013;1(1):35-43.

18. Candioti LV, DeZan MM, Cámara MS, Goicoechea HC. Experimental design and multiple response optimization using the desirability function in analytical methods development. Talanta. 2014;124:123-38. doi: 10.1016/j. talanta.2014.01.034.

19. Venkanna A, Siva B, Poornima B, Vadaparthi PR, Prasad KR, Reddy KA, et al. Phytochemical investigation of sesquiterpenes from the fruits of Schisandra chinensis and their cytotoxic activity. Fitoterapia. 2014;95:102-8.

20. Gomes EC, Mussel WN, Resende JM, Fialho SL, Barbosa J, Carignani E, et al. Yoshida MI. Characterization of tenofovir disoproxil fumarate and its behavior under heating. Cryst Growth Des. 2015;15(4):1915-22.

21. Shailender J, Ravi PR, Saha P, Dalvi A, Myneni S. Tenofovir disoproxil fumarate loaded PLGA nanoparticles for enhanced oral absorption: Effect of experimental variables and in vitro, ex vivo and in vivo evaluation. Colloids Surf B Biointerfaces. 2017;158:610-9.

22. Yang X, Trinh HM, Agrahari V, Sheng Y, Pal D, Mitra AK. Nanoparticle-based topical ophthalmic gel formulation for sustained release of hydrocortisone butyrate. AAPS PharmSciTech. 2016;17(2):294-306.

23. Hasan S, Kumar N, Bakan JA. Microencapsulation. The theory and practice of industrial pharmacy. New Delhi: CBS Publishers. 2014;579-96.

24. Budhian A, Siegel SJ, Winey KI. Production of haloperidol-loaded PLGA nanoparticles for extended controlled drug release of haloperidol. J Microencapsul. 2005;22(7):773-85.

25. Krishnamachari $\mathrm{Y}$, Madan $\mathrm{P}$, Lin S. Development of $\mathrm{pH}$-and time-dependent oral microparticles to optimize budesonide delivery to ileum and colon. Int $\mathrm{J}$ Pharm. 2007;338(1-2):238-47.

26. Gupta B, Poudel BK, Pathak S, Tak JW, Lee HH, Jeong JH, et al. Effects of formulation variables on the particle size and drug encapsulation of imatinibloaded solid lipid nanoparticles. AAPS Pharm Sci Tech. 2016;17(3):652-62.

27. Nicolete R, DosSantos DF, Faccioli LH. The uptake of PLGA micro or nanoparticles by macrophages provokes distinct in vitro inflammatory response. Int Immunopharmacol. 2011;11(10):1557-63.

28. Gorner T, Gref R, Michenot D, Sommer F, Tran MN, Dellacherie E. Lidocaineloaded biodegradable nanospheres. I. Optimization of the drug incorporation into the polymer matrix. J Control Release. 1999;57(3):259-68.

29. Sharma N, Madan P, Lin S. Effect of process and formulation variables on the preparation of parenteral paclitaxel-loaded biodegradable polymeric nanoparticles: A co-surfactant study. Asian J Pharm Sci. 2016;11(3):404-16.

30. Brillo J, Pommrich Al, Meyer A. Relation between self-diffusion and viscosity in dense liquids: New experimental results from electrostatic levitation. Phys Rev Lett. 2011;107(16):165902.

31. Dördelmann G, Kozlova D, Karczewski S, Lizio R, Knauer S, Epple M. Calcium phosphate increases the encapsulation efficiency of hydrophilic drugs (proteins, nucleic acids) into poly (d, I-lactide-co-glycolide acid) nanoparticles for intracellular delivery. J Mater Chem B. 2014;2(41):7250-9.

32. Kashi TS, Eskandarion S, Esfandyari-Manesh M, Marashi SM, Samadi N, Fatemi SM, et al. Improved drug loading and antibacterial activity of minocycline-loaded PLGA nanoparticles prepared by solid/oil/water ion pairing method. Int J Nanomedicine. 2012;7:221-34.

33. Munekata T, Suzuki T, Yamakawa S, Asahi R. Effects of viscosity, surface tension and evaporation rate of solvent on dry colloidal structures: A lattice Boltzmann study. Phys Rev E. 2013;88(5):052314.

34. Stojak M, Mazur L, Opydo-Chanek M, Łukawska M, Oszczapowicz I. Effects of structural modifications of daunorubicin on in vitro antileukemic activity. Anticancer Res. 2012;32(12):5271-7. 
PICTORIAL ABSTRACT

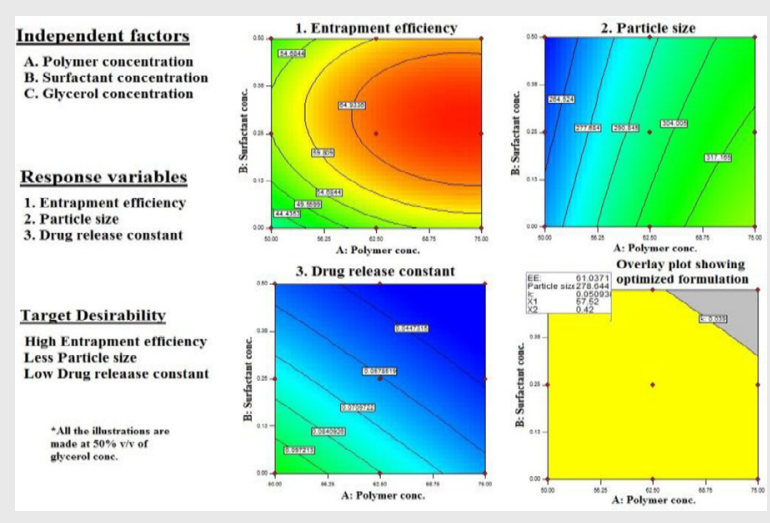

\section{SUMMARY}

Optimization using statistical techniques and software for formulation of PLGA nanoparticles loaded with TDF was performed in this work. PLGA-TDF-NCs were prepared by modified multiple emulsion $(\mathrm{w} / \mathrm{o} / \mathrm{w})$ technique. Polymer concentration, surfactant concentration in the secondary emulsion and composition of outermost phase were selected as the independent variables. Entrapment efficiency, particle size and drug release constant were taken as response variables. The experiment was designed as a $3^{3}$ factorial design under RSM. The prepared formulations were evaluated for the response variables. The obtained results were statistically treated by response surface polynomial quadratic model using Stat Ease design expert software. The selected model and influences of all the factors on responses were found to be significant. Later, optimization was performed using desirability functions approach with the target of high entrapment efficiency, less particle size and low drug release constant. The optimized formulation was obtained with maximum entrapment efficiency of $63.08 \%$; particle size of $284.53 \mathrm{~nm}$ and $0.054 \mathrm{~h}^{-1}$ of drug release constant, thus indicating the set objectives of the work were successfully achieved.

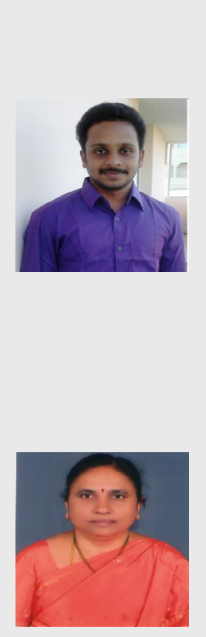

\begin{abstract}
About Authors
Grandhi Srikar He has been working as an Assistant Professor since August 2012 in University College of Pharmaceutical Sciences, Acharya Nagarjuna University, Guntur, India. He has more than 10 years of academic and research experience. He has done his masters degree in Pharmaceutics specialization. Now, he is carrying out his research work on biodegradable nanoparticles with improved entrapment efficiency of aqueous soluble drugs and aimed for liver targeting. Besides, he is working on development of new technologies to achieve zero order drug release from oral controlled drug delivery systems and also on development of co-processed excipients.
\end{abstract}

Avula Prameela Rani She is working as Principal and Professor in University College of Pharmaceutical Sciences, Acharya Nagarjuna University, Guntur, India. She has more than 25 years of teaching and research experience in various positions. She has guided 15 students to complete their Ph.D degrees. She has published more than 150 research and review articles in various national and international peer reviewed journals. She has gotten three funded projects as principal investigator by UGC, AICTE and DST for a total fund of Rs. 85 lakhs. Recently she has been awarded with Best Researcher of the Year award - 2019 by A.P Science City, Govt. of Andhra Pradesh.

Cite this article: Srikar G, Rani AP. Tenofovir Loaded Poly (Lactide-Co-Glycolide) Nanocapsules: Formulation Optimization by Desirability Functions Approach. Indian $\mathrm{J}$ of Pharmaceutical Education and Research. $2020 ; 54(2 s): s 230-s 240$. 